\title{
Neo-MDF: Material Data Form
}

\section{OLIVER POPADICH}

University of Michigan

Anthropologist Tim Ingold offers a description of material in three parts - medium, substance and surface. ${ }^{1}$ Using this framework, computer scientist and anthropologist Paul Dourish unpacks one way in which data can be considered material: if data is the substance and software is the medium, then the surface is the software's representation of this information. ${ }^{2}$

In other words, we perceive the representation of data as a surface. ${ }^{3}$ However, being a virtual entity, the material data must manifest physically as part of the surface of a physical material (as light, print, toolpath, etc.), thus becoming embedded within the qualities of that material. From truss to trim, modern surfaces depend on data and the management of said data, can motivate the appearance of architecture in new and unexpected ways. ${ }^{4}$

This project posits that contemporary materials are not a single entity, rather a combination of physical and virtual qualities that manifest as a hybrid-material. These hybrid-materials are palpable, driving a phenomenological experience ultimately derived from their digital underpinnings.

The proposed building is a fusion between library and data center - an embodiment of the history and evolution of our production, storage and consumption of information. Bisecting these programs are the distinct ceiling planes, produced through displacement and normal mapping - rendering processes that imbue surfaces with material qualities like texture and form. By working within these rendering methodologies the project contends with the layered relationship between data and material and their physical formation as objects.

It becomes clear that the world around us is designed for and by data. Some things, like the ceilings and trusses are designed as translations of data into form; others, like cables and screens are designed to transmit data from place to place. As the concept of "glitch" become materialized and the mutability of information is questioned, hybrid-materials put new pressure on our understandings of temporality, presence and separation, ultimately, redefining the roles and objectives of designers.

\section{ENDNOTES}

1 Tim Ingold, "Materials against materiality," Archaeological Dialogues 14, no. 1 (2007). A medium, like air, is the ethereal matter that enables movement and perception; a substance, like wood, is the physical thing, opaque and dense. Between these two poles is surface - the component that defines pattern, texture, shape and generally all perceivable qualities about a material.

2 Paul Dourish, The Stuff of Bits: An Essay on the Materialities of Information (Cambridge, MA: The MIT Press, 2017).

3 John May, "Everything is Already an Image," Log 40 (2017): 9-26.

4 Jack Burnham, "Systems Esthetics," Artforum (September 1968). "Scientists and technicians are not converted into 'artists,' rather the artist becomes a symptom of the schism between art and technics." Fifty years later, the hybrid-material reveals that their former split concluded as a union between art and technics. The resulting environment has been converted into a landscape of digitally imbued information. 




